\title{
The theory of latent vulnerability: Reconceptualizing the link between childhood maltreatment and psychiatric disorder
}

\author{
EAMON J. MCCRORY AND ESSI VIDING \\ University College London
}

\begin{abstract}
Maltreatment in childhood is associated with a significantly increased likelihood of psychiatric disorder that endures across the life span. If disorders emerge they tend to be more severe and less responsive to treatment. We introduce the concept of latent vulnerability as a way of conceptualizing the nature of this psychiatric risk. We argue that vulnerability to mental health problems can be understood as changes in a suite of neurocognitive systems that reflect adaptation or altered calibration to early neglectful or maltreating environments. Altered threat processing is presented as one exemplar candidate system. Heightened neurocognitive vigilance to threat is argued to reflect a calibration to an early at-risk environment that becomes maladaptive (and instantiates vulnerability) in the longer term. Other neurocognitive domains, including reward and memory processing, represent equally promising candidates for indexing latent vulnerability and warrant future enquiry. We suggest that the operationalization of latent vulnerability has the potential to guide a preventative psychiatry approach. Intervention currently occurs at two stages when maltreatment is confirmed: first, by addressing issues of risk; and second, by providing clinical intervention if a child meets criteria for psychiatric disorder. We argue that indexing latent vulnerability represents a third intervention opportunity, with the potential to target an indicated prevention approach for the most vulnerable children, offsetting risk trajectories before psychiatric disorders emerge.
\end{abstract}

There is now an overwhelming body of evidence that childhood maltreatment significantly increases the risk of psychiatric disorder in adolescence and throughout adulthood (Gilbert et al., 2009). It is important to note that such an association is probabilistic: maltreatment experience serves to significantly increase the likelihood of poor outcome (e.g., Koenen \& Widom, 2009; Widom, DuMont, \& Czaja, 2007). What is remarkable is the sheer diversity of disorders that are potentiated by exposure to this form of early adversity. These range from depression (Anda et al., 2002), anxiety (Scott, Smith, \& Ellis, 2010), and borderline personality disorder (Widom, Czaja, \& Paris, 2009) to schizophrenia (Read, Os, Morrison, \& Ross, 2005). This impact on psychological health is in turn embedded within a broader spectrum of maladaptive outcomes associated with maltreatment, which includes poorer physical health (Widom, Czaja, Bentley, \& Johnson, 2012), economic productivity (Currie \& Widom, 2010), educational attainment, and social functioning (Nikulina, Widom, \& Czaja, 2011). This spectrum of difficulties suggests that maltreatment (i.e., experiences of neglect and/or physical, sexual, and emotional abuse) leaves its mark by altering core aspects of functioning that are likely to play a general role in the ability to successfully negotiate normative stresses and developmental challenges across the life span.

The preparation of this article was supported by the UK Economic and Social Research Council (Grant ES/K005723).

Address correspondence and reprint requests to: Eamon McCrory, Developmental Risk and Resilience Unit, 26 Bedford Way, University College London WC1H 0AP, UK; E-mail: e.mccrory@ucl.ac.uk.
If this picture were not sufficiently sobering, an emerging body of research suggests that when a psychiatric condition does present in an individual who has experienced childhood maltreatment, the nature of the individual's disorder is more problematic or intractable in a number of respects. First, psychiatric disorders in individuals who have experienced maltreatment are likely to develop earlier, and with more severe symptomatology (Hovens et al., 2010). Second, such disorders are more likely to present alongside other co-occurring conditions (Harkness \& Wildes, 2002). This increased likelihood of co-morbidity is consistent with the general supposition that the experience of maltreatment has a broad rather than focal impact on functioning. Third, a disorder in an individual who has experienced childhood maltreatment is more likely to be persistent and recurrent and less likely to respond to standard treatment approaches (Hovens et al., 2012; Nanni, Uher, \& Danese, 2012). Studies have shown that both psychological and pharmacological interventions for depression (for example) are less effective for individuals with prior experiences of childhood maltreatment (Harkness, Bagby, \& Kennedy, 2012; Nanni et al., 2012).

Children who have experienced such adversity can therefore be understood to represent a particularly vulnerable group at elevated risk of mental health difficulties. The approach of social and healthcare providers to the substantial challenge posed by maltreatment has involved prevention as well as social care and mental health intervention following maltreatment experience. There has been an important and necessary emphasis on what has been termed primary prevention. For example, programs such as the well-evaluated 
Nurse Family Partnership program have been shown to reduce cases of child abuse and neglect even after a 15-year follow-up (Olds et al., 1997). However, despite the significant success of this and other prevention programs, there continue to be a huge number of children about whom professionals have concerns. In 2012, referrals regarding maltreatment were made in relation to 6.3 million children in the United States alone (US Department of Health and Human Services, 2013). Of these, 686,000 cases were substantiated. Once maltreatment has been confirmed, the question arises: how can we act to help and support these children? There are typically two intervention stages following experience of maltreatment. First, there is an appropriate focus on addressing child protection and welfare concerns, ensuring that children are safe and if necessary moved into an alternative setting. Second, there are mental health interventions, but typically only for those children who meet psychiatric diagnostic criteria. While there are some initiatives to support children and families, including support for foster care providers, it is unusual for children who experience maltreatment to receive any specialist input or preventative intervention in relation to mental health until they meet criteria for a psychiatric disorder, when they are referred, like any other child, to a generic clinic for disorder-specific treatment. While the prior experience of maltreatment may be a pertinent factor in clinical formulation, it does not usually represent a primary organizing principle in the design of the intervention.

We believe that this current approach is failing many children. Even though we know maltreatment is associated with significantly elevated risk of psychiatric disorder, the received wisdom is to wait until full-blown disorders emerge before treatment is offered. Such an approach is increasingly at odds with a recent shift toward preemptive psychiatry (e.g., Insel, 2009; McGorry, 2013). Given the nature of the known vulnerability associated with this group of children, the challenge is surely to identify these individuals, and offset their risk trajectory, by providing a form of preventative intervention that can reduce the likelihood of a psychiatric illness in the future.

In this paper, we outline the concept of latent vulnerability as an important organizing construct that has the potential to inform such a strategy. Below we develop our definition of latent vulnerability and then systematically consider altered threat processing as one potential candidate of latent vulnerability. Finally, we explore the translational implications and challenges of operationalizing this construct in a clinical context.

\section{The Theory of Latent Vulnerability}

Latent refers to a quality or state existing but not yet developed or manifest. We suggest that there are measurable changes that follow from experiences of maltreatment that are latent in so far as they do not necessarily have an immediate clinical manifestation, but nonetheless are associated with an increased risk of, or vulnerability to, future psychiatric dis- order. An index of latent vulnerability therefore captures the degree to which an ostensibly healthy individual previously exposed to maltreatment is at future risk of developing a psychiatric disorder. A significant degree of latent vulnerability is likely to characterize a proportion of children who experience maltreatment and neglect, but currently we lack the ability to differentiate these children, or make assessments regarding their relative degree of latent vulnerability. In order to make explicit the nature of this construct, we draw out four key features that are central to its definition and relevant to its operationalization.

\section{Beyond symptomatology}

There is now a growing literature in the field of psychosis research delineating how preclinical symptomatology can constitute a prodromal presentation that occurs before the onset of a psychotic episode (e.g., McGlashan \& Johannessen, 1996). The prodrome phase includes both generic symptoms, for example, changes in irritability or depression, and attenuated positive symptoms, such as thought disorder or ideas of reference. Such prodromal symptoms and markers of latent vulnerability share a number of features: both are associated with an increased risk of psychiatric disorder; both may be manifest at psychological and behavioral levels; and both may have an impact on functioning independently of the presence or absence of a disorder. However, these concepts differ in a number of important respects. Markers of latent vulnerability are not necessarily symptoms of any future disorder. They may refer, for example, to cognitive processes or representations that increase the likelihood that a set of symptoms may develop, but these symptoms may be qualitatively different. For example, as we discuss later, an increased tendency to overgeneral autobiographical memory functioning may increase vulnerability to depression (Rawal \& Rice, 2012), but this pattern of memory functioning does not constitute a symptom of depression.

\section{A systems-level index}

Our view is that latent vulnerability in maltreated individuals is best indexed by a systems-level approach. In other words, it is a complex phenotype that can be thought of as a "maladaptive calibration" in one or more systems important for socioemotional and cognitive functioning. Understanding the way in which latent vulnerability emerges following maltreatment may be achieved in theory by focusing on different levels of analyses. For example, one might investigate how genetic, cellular, or hormonal factors contribute to the developmental emergence of a complex, system-level latent vulnerability phenotype. Such work is critical if we are to elucidate causal mechanisms. For example, we may be interested in how people with different genotypes respond to maltreatment experience and how this influences their gene expression and hypothalamic-pituitary-adrenal axis functioning over time, resulting in an overvigilance to threat and biased processing 
of ambiguous information (seen as threatening). This multilevel approach is consistent with the dominant framework of developmental psychopathology and resilience research (Cicchetti, 2013; Cicchetti \& Bender, 2006; Cicchetti \& Toth, 2009) as well as a key tenet of the National Institute of Mental Health's Research Domain Criteria initiative (Insel, 2014; Insel et al., 2010). Each of these approaches proposes that risk for mental disorder is best indexed by considering a range of system-level dimensional constructs that are essentially agnostic about disorder categories.

However, the question of how we best capture or "diagnostically index" latent vulnerability is a related but distinct issue from the investigation of how latent vulnerability develops. We suggest that from a translational perspective, how we accurately quantify atypical "system calibration" denoting increased risk for psychiatric disorder is of crucial importance in its own right and is best operationalized at the neurocognitive systems level. Given the heterogeneity of maladaptive outcomes associated with maltreatment across psychiatric disorders (Gilbert et al., 2009), it would be reasonable to hypothesize that a limited but varied set of candidate neurocognitive systems are compromised or shaped in a way that increases psychiatric vulnerability following trauma exposure.
An embedded vulnerability factor

We propose that latent vulnerability is present and can be indexed prior to onset of psychiatric disorder. However, that latent vulnerability is present does not necessarily inform us as to the timing of disease onset; such vulnerability could theoretically be present for months or years, but clinical symptoms (both behavioral and cognitive) may only manifest under certain conditions characterized by stress or challenge or may never manifest given adequate intrinsic and extrinsic protective factors, favorable conditions, and resilient genotypes, despite the enduring presence of latent vulnerability (see Figure 1). In the same way that a set of hidden structural deficits in a building, for example, may only become manifest under severe weather conditions, so too might the inherent weaknesses associated with elevated latent vulnerability only manifest as clinical symptoms following stress exposure. This concept is different from how the idea of "prodrome" is typically used in the field of psychosis research, denoting a period of time, which occurs prior to an individual developing a psychotic episode, during which a pattern of behavioral and cognitive changes is manifest (e.g., Yung \& McGorry, 1996).

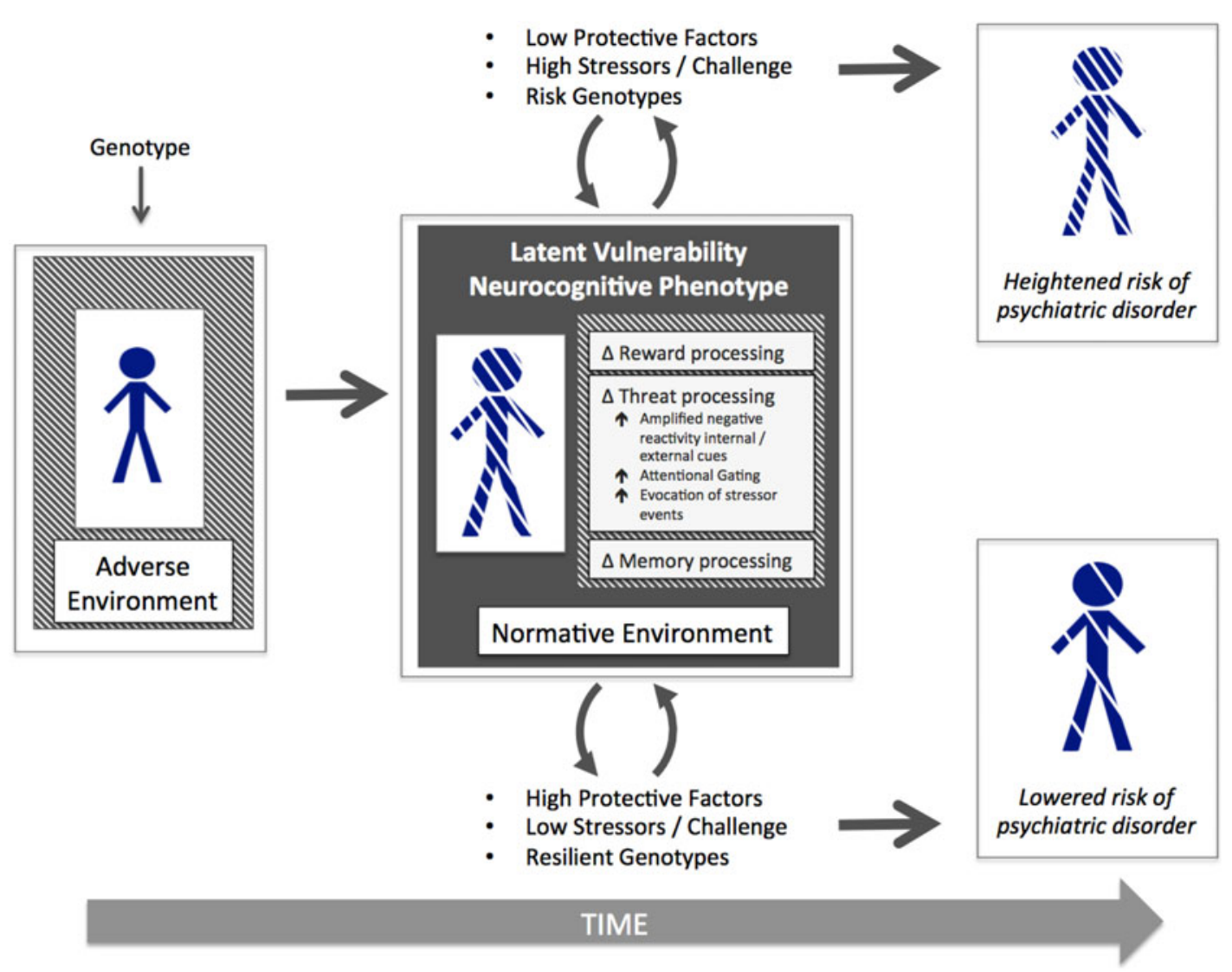

Figure 1. (Color online) A schematic displaying the embedding of latent vulnerability at the neurocognitive level and differential outcome in relation to psychiatric risk depending on protective factors, stressor exposure, and genotypes. 


\section{Predictive validity}

A true marker of latent vulnerability must have a predictive value in signaling heightened risk of future psychiatric disorder in individuals who have experienced maltreatment among a group who are already at elevated risk of psychopathology. Thus, although we might be able to identify a range of neurocognitive correlates of maltreatment experience, any given correlate could only be described as a marker of latent vulnerability if individual differences in this factor significantly contribute to our estimation of individual differences of future psychiatric risk.

Here we consider one system-level construct that represents a promising candidate marker of latent vulnerability: threat processing. We focus our discussion on evidence pertaining to the neurocognitive level, because this level of analysis can capture system-level processes (and supporting neural architecture) that bias an individual's computations about the world. The computational processes instantiated at the neurocognitive level represent the developmental product of multiple and dynamic genetic, epigenetic, and environmental processes. As such, the functioning of a given system at the neurocognitive level will be to a degree congruent with, and will have resulted from, adaptation to a nonnormative environment characterized by threat, deprivation, or unpredictability. However, such adaptation to environmentspecific contingencies may render the system less well optimized for more typical environments with different processing demands.

The question arises as to what evidence would be required to establish the viability of a putative construct of latent vulnerability. We suggest that at least three core criteria need to be met. Specifically, it should be possible to demonstrate that any system-level construct can be shown to be the following:

1. associated with maltreatment experience, even in the absence of (or prior to) the development of psychiatric disorder;

2. predictive of individual differences in psychiatric outcome among individuals who have experienced maltreatment in childhood; and

3. associated with relevant psychiatric disorders known to be elevated among individuals who have experienced maltreatment.

We now review the current evidence pertaining to threat processing in relation to these three criteria.

\section{Threat Processing: A Candidate System Indexing Latent Vulnerability}

\section{Threat processing and its neural bases}

Survival of an organism crucially depends on the ability to accurately detect stimuli associated with danger and respond in ways that serve to mitigate such threat. There is a rich and longstanding neurocognitive literature, including studies of typical adults and maltreated children, demonstrating that threat-related stimuli are prioritized for processing (e.g., LeDoux, 2000; Öhman, 2009; Rieder \& Cicchetti, 1989).

Much of what we know about the neural processes that underpin our ability to process threat has been informed by decades of animal research. The amygdala, a heterogeneous medial temporal lobe structure composed of a varied collection of interconnected subnuclei, has been shown to play a privileged role in fear learning and threat detection; however, there is increased recognition that it is also involved in other social cognitive and motivational processes, and concerned with salient environmental cues more generally. The amygdala receives extensive afferent projections from the thalamus as well as sensory and association cortices (Pape \& Pare, 2010) and in turn projects a widespread set of efferents to both subcortical structures (notably the hypothalamus, the bed nuceli of stria terminalis, periaqueductal gray, and striatum) as well as a set of cortical structures with which the amygdala is reciprocally connected (including the medial prefrontal cortex, insula, and sensory cortices). Human lesion studies have demonstrated a high degree of consistency in functionality of this fear network, indicating that the amygdala plays a critical role in learning and initiating conditioned responses (LaBar, LeDoux, Sencer, \& Phelps, 1995). It should be emphasized that amygdala reactivity to threat reflects only one component of a distributed processing network, encompassing the medial prefrontal cortex (and the anterior cingulate cortex in particular), as well as the insula (e.g., Etkin \& Wager, 2007; Kerestes, Davey, Stephanou, Whittle, \& Harrison, 2013; Shin \& Liberzon, 2010). For example, the amygdala plays a critical role in fear extinction (where an individual ceases to regard a given stimulus as threatening) along with several other brain areas (in particular the ventromedial prefrontal cortex; Milad \& Quirk, 2002). As we discuss later, there is emerging evidence that this neurocognitive system appears to recalibrate or adapt in children and in adults following sustained exposure to environmental danger.

\section{Threat processing evaluated against the three core criteria}

What is the evidence that maltreatment experience is associated with atypical threat processing, even in the absence of, or prior to, the development of psychiatric disorder? Findings from a range of methodologies suggest atypical processing of threat cues in children exposed to maltreatment. In a series of psychological studies Pollak and colleagues have shown that children exposed to physical abuse have broader perceptual boundaries for categorizing anger (Pollak \& Kistler, 2002) and can accurately identify angry facial expressions using sparse perceptual information (Pollak, Messner, Kistler, \& Cohn, 2009; Pollak \& Sinha, 2002). These findings have been supported by EEG studies that have demonstrated atypical processing of angry faces at the neural level in school-aged children exposed to maltreatment. For example, 
relative increases in amplitude of the $\mathrm{P} 3 \mathrm{~b}$ event-related potential (ERP) component have been reported when physically abused children actively search for or attend to angry faces (Pollak, Cicchetti, Klorman, \& Brumaghim, 1997; Pollak, Klorman, Thatcher, \& Cicchetti, 2001), suggesting hyperresponsiveness to angry facial expressions relative to nonmaltreated children. In addition, P3b amplitude has been found to correlate with symptoms of anxiety in children who have experienced physical abuse (Shackman, Shackman, \& Pollak, 2007).

Cicchetti and colleagues have even demonstrated alterations in ERP responses in much younger children exposed to maltreatment, reporting altered ERP response to facial affect in children at 30 months and 42 months of age who had experienced maltreatment in the first 12 months of life (Cicchetti \& Curtis, 2005; Curtis \& Cicchetti, 2011). More recently, Curtis and Cicchetti (2013) examined the neural correlates of facial affect processing in 15-month-old infants who had documented experiences of maltreatment before the age of 12 months. Even at this young age, an altered ERP pattern to facial expressions was observed for both angry and happy faces in maltreated compared to nonmaltreated infants. Collectively, these studies using both behavioral and electrophysiological indices provide convincing evidence that children who have been exposed to maltreatment show preferential attention for (or difficulty disengaging from) human cues conveying threat.

In recent years, several functional magnetic resonance imaging (fMRI) studies have been conducted, complementing these psychological and electrophysiological studies. These have consistently reported heightened amygdala reactivity to threat-related cues in children exposed to early adversity or childhood maltreatment. In two fMRI studies using emotional face-processing paradigms, children who had experienced early institutionalization were found to exhibit increased amygdala response to threatening facial cues (Maheu et al., 2010; Tottenham et al., 2011). We have reported similar findings in two studies of children exposed to maltreatment at home, in community settings (McCrory et al., 2011, 2013). In one study, we asked children exposed to documented maltreatment $(n=20)$ and a matched group of community peers $(n=23)$ to decide the gender of a series of faces that varied in emotional expression (neutral, angry, and sad). Children exposed to maltreatment were found to show significantly increased activation in the anterior insula and amygdala in response to angry but not sad faces. The children exposed to maltreatment did not present with significantly elevated levels of anxiety or depression, suggesting that the findings were not secondary to these psychiatric disorders. An exploratory analysis also found that the mean contrast estimates within the left anterior insula positively correlated with a measure of violence exposure at home. This dose-response relationship may reflect a calibration of the threat-related value of anger cues in the maltreated children, consistent with the role of the anterior insula in the integration of pain anticipation and salience (Wiech et al., 2010).

In a subsequent study with a related sample, we investigated the neural responses to preattentively presented facial cues (neutral, angry, and happy) using a masked dot-probe paradigm (McCrory et al., 2013). As predicted, children exposed to maltreatment at home $(n=18)$, compared to a group of matched peers $(n=23)$, were found to show significantly elevated amygdala activation to angry compared to neutral faces, even without conscious awareness of having viewed facial stimuli. In addition, amygdala reactivity in maltreated children compared to nonmaltreated peers was positively correlated with the age of onset and duration of emotional abuse. These associations between maltreatment severity and brain response are in line with the notion that threat exposure recalibrates neural circuitry involved in threat reactivity. Contrary to our predictions, however, the maltreated group also showed significantly elevated amygdala activation to happy compared to neutral faces. It may be that during early stages of affect processing, prior to the engagement of higher order regulatory systems, maltreatment experience is associated with increased neural responsiveness to heightened affect/ salience in general, with a selective response to threat characterizing only later stage of processing. This may be adaptive if displays of negative or positive affect in a home characterized by threat and violence signal behavioral unpredictability. Alternatively, the increased amygdala activation observed for happy faces may represent a heightened response to relatively infrequent positive social stimuli, pointing to atypical processing of social reward. Future studies are required to investigate these possibilities further.

In a large sample of typically developing adolescents $(n=139)$, White et al. (2012) investigated whether geneenvironment interaction effects were related to individual differences in threat-related activity of the amygdala. Elevated self-report assessment of emotional neglect was related to increased amygdala reactivity, but only in individuals carrying risk polymorphisms of the FK506 binding protein 5 (FKBP5) gene. These risk variants of the FKBP5 gene had previously been associated with reduced downregulation of hypothalamic-pituitary-adrenal axis activity following stress and/or stress-related psychopathology in animal and human studies.

These findings from studies of children and adolescents who had experienced maltreatment are consistent with studies investigating heightened responsiveness of the amygdala in adults reporting historic experiences of childhood abuse. Dannlowski et al. (2012) employed an emotional face-matching paradigm to probe amygdala reactivity to threat-related faces in a large community sample $(n=148)$, where childhood maltreatment experiences were assessed via self-report. A robust positive association was found between overall maltreatment scores and amygdala responsiveness to threatrelated facial expressions. This association was not influenced by trait anxiety, depression level, age, intelligence, education, or more recent stressful life events. A subsequent study employing a subliminal priming paradigm with sad, neutral, and happy faces, again in a community sample $(n=150)$ of adults free from any prior history of psychiatric disorder (Dannlowski et al., 2013), found that maltreatment scores were positively associated with amygdala responsiveness in 
relation to sad, but not happy facial expressions. A similar pattern of findings were reported by Grant, Cannistraci, Hollon, Gore, and Shelton (2011) in a group of clinically depressed patients $(n=20)$ using a facial flanker task, where level of childhood physical abuse correlated positively with right amygdala reactivity to sad versus neutral faces.

In summary, the evidence from psychological, electrophysiological, and neuroimaging paradigms have indicated a consistent pattern of atypical processing of threat-related cues in children who have experienced maltreatment, and adults who report historical experiences of abuse. Specifically, maltreatment appears to be associated with increased hypervigilance to threat, and potentially salience more generally, indexed by greater amygdala reactivity, which in turn appears to be associated with the level of maltreatment severity experienced (McCrory et al., 2013; Dannlowski et al., 2012, 2013).

Is threat processing predictive of individual differences in psychiatric outcome among individuals who have experienced maltreatment? One cross-sectional study has reported an association between P3b amplitudes to maternal vocal anger and anxiety symptoms in children, and found that this measure of attentional allocation to threat statistically mediated the relationship between abuse experience and children's anxiety symptoms (Shackman et al., 2007). However, to our knowledge, there have been no longitudinal studies that have investigated the association between threat processing and subsequent psychiatric symptomatology in individuals who have experienced maltreatment. As we discuss in more detail later, such studies have been conducted in populations of soldiers before and after exposure to combat; longitudinal studies of children are urgently needed.

Is altered threat processing associated with relevant psychiatric disorders known to be elevated among individuals who have experienced maltreatment? Threat processing and amygdala function have been extensively investigated in clinical samples of adolescents and adults at risk of anxiety and depression, as well as in those who already meet clinical diagnostic criteria. The findings have been relatively consistent, with increased amygdala and insula reactivity associated both with elevated risk of mood and anxiety disorders (Stein, Simmons, Feinstein, \& Paulus, 2007; Wolfensberger, Veltman, Hoogendijk, Boomsma, \& de Geus, 2008) and as features of clinical presentation (e.g., Etkin \& Wager, 2007; Fales et al., 2008; Shin \& Liberzon, 2010). For example, heightened amygdala reactivity has been observed when adult patients with anxiety and posttraumatic stress disorder (PTSD) consciously process threat-related stimuli such as trauma-related narratives or photographs (Liberzon \& Sripada, 2007; Liberzon et al., 1999) or more generic stimuli such as fearful facial expressions relative to healthy controls (Etkin \& Wager, 2007). At the behavioral level, anxious individuals are commonly reported to show a greater attentional bias to threat compared to nonanxious controls (Mogg \& Bradley, 1998), with findings in anxious children comparable to those seen in adults (e.g., Roy et al., 2008), although this pattern is not always consistent (e.g., Pine et al., 2005).

\section{Threat processing as an index of latent vulnerability: Evidence from other populations}

Given the paucity of functional neuroimaging studies in the maltreatment field at the current time, we here cast a wider net across the literature to consider whether the study of other stress-exposed populations can help inform the evaluation of altered threat processing as an index of latent vulnerability. For several reasons, the study of soldiers exposed to combat is an exceptionally helpful parallel literature in this regard.

First, a wealth of studies has investigated the relationship between the mental health of military personnel and stress exposure in the field immediately on return from combat, and over much longer time periods. We now know that individuals exposed to combat exposure have a two- to threefold increased risk of developing mental health problems, including PTSD, compared to individuals not exposed to combat, even if they do not initially present with a mental health problem (Thomas et al., 2010), analogous to elevated risks seen in children exposed to maltreatment. Although the forms of environmental stress posed by a maltreating home environment and a combat field differ significantly, both are associated with a marked elevation in psychiatric risk.

Second, the study of soldiers provides the opportunity to conduct longitudinal investigations capable of measuring behavioral and neural function before and after stress exposure in ways that would be entirely unethical with child samples. Such studies can allow us to more confidently make inferences about adaption following stress, reducing the likelihood that neurocognitive correlates associated with stress exposure were present prior to the stressor event. For example, van Wingen, Geuze, Vermetten, and Fernández, (2011) have demonstrated that stress exposure incurred following combat alters the functional properties of both the amygdala and anterior insula (see also Admon et al., 2009, 2013). Both regions showed increased functional reactivity in soldiers to threat cues (angry and fearful faces) approximately 1.5 months postcombat, compared to that seen during precombat scans (van Wingen et al., 2011). This pattern of increased activation in the amygdala and anterior insula during conscious threat processing in soldiers is strikingly similar to what we observed in maltreated children (McCrory et al., 2011), suggesting that the heightened reactivity of these areas to threat cues are not reflective of "damage," but in both groups may represent a pattern of adaptation to environmental threat.

Third, studies of military personnel are particularly well suited to prospective designs, which provide an opportunity to investigate whether preexisting individual differences prior to combat can predict future psychiatric disorder. Analogous studies are not ethically possible with children, for whom the first priority is always the protection from future harm. Specifically, it is possible in studies of combat exposure to assess whether individual differences in threat reactivity prior to 
stress exposure are associated with the likelihood of future symptomatology. Within the psychological literature, it has been reported that the greater the intensity of the initial emotional reaction to a stressful event, the greater the likelihood of subsequent mental health problems, including PTSD (Creamer, McFarlane, \& Burgess, 2005; Ozer, Best, Lipsey, $\&$ Weiss, 2003). This suggests that individual differences in threat-related emotional reactivity/modulation may at least in part explain the variation in psychiatric outcome seen in individuals exposed to stress.

In line with these findings, two seminal prospective neuroimaging studies by Admon et al. $(2009,2013)$ investigated threat reactivity prior to combat and its relationship to subsequent PTSD symptomatology. In the first study, newly recruited healthy soldiers $(n=50)$ were assessed at two time points: during initial training, prior to stress, and approximately 18 months later, following combat deployment after stress (Admon et al., 2009). At each time point, PTSD symptoms were assessed and brain responses to threatening (pictures of military content) and neutral stimuli (pictures of civilian content) were measured using fMRI. As predicted, stress-related behavioral symptoms increased following stress exposure, as did amygdala reactivity. More surprising, the level of amygdala activation prior to stress exposure significantly correlated with the degree to which PTSD symptoms increased after stress exposure. This study provided the first direct evidence indicating a relationship between baseline amygdala reactivity (predating stress) and the tendency of an individual to develop PTSD symptoms (following subsequent stress exposure). The variability in amygdala reactivity before stress explained almost $50 \%$ of the variance in the increase in symptoms observed following field exposure.

In the second study, a smaller sample of soldiers $(n=24)$ were scanned prior to military service and following exposure to military service during a competitive game task that allowed assessment of risky and safe anticipation as well as rewarding or punishing outcomes (Admon et al., 2013). Admon et al. focused on reward and risk-related regions, including the amygdala. They found that greater amygdala activation in response to risky anticipation preexposure was significantly associated with the level of postexposure PTSD symptoms. This suggests not only that amygdala responsiveness increases following combat exposure (Admon et al., 2009; van Wingen et al., 2011) but also that greater amygdala responsiveness to aversive/threat-related stimuli constitute a preexisting vulnerability factor that increases the likelihood of psychopathology symptoms following exposure to stress.

On the basis of these prospective studies of military personnel, we can draw several conclusions pertinent to the field of maltreatment research. First, exposure to significant environmental threat is associated with an enduring elevation of psychiatric risk. Second, amygdala reactivity to threat cues is heightened following exposure to environments characterized by biological threat. Such a response is similar to what is observed in animal studies, which have shown that severe stress can sensitize amygdala responsivity and also reduce the degree of regulation prefrontal regions (Correll, Rosenkranz, \& Grace, 2005; Rosenkranz, Venheim, \& Padival, 2010). Third, these studies indicate that baseline level of amygdala reactivity prior to combat exposure predicts subsequent levels of symptomatology following stress exposure. This pattern of findings would be compatible with the notion that in some adult soldiers, preexisting patterns of heightened amygdala responsiveness to threat-related cues may index a degree of latent vulnerability. One might speculate, for example, whether these individual differences are, at least in part, shaped by exposure to early adversity and childhood maltreatment? This question warrants further investigation.

\section{Summary: Threat processing-A system level candidate for latent vulnerability?}

We suggest that an emerging body of evidence points to altered threat reactivity, as indexed in part by heightened neural response of the amygdala (and related structures) to biological threat stimuli, as one potential system level candidate for latent vulnerability in children exposed to maltreatment. Three lines of evidence support this contention.

First, we know that severity of maltreatment experience appears to be associated with greater levels of amygdala reactivity to threat (Dannlowski et al., 2012, 2013; McCrory et al., 2013), although this relationship is almost certainly moderated by genotype (e.g., White et al., 2012). At the neural level, combat exposure, a very different kind of environmental danger, has been associated with increased reactivity of the amygdala and anterior insula in longitudinal designs, suggesting that these changes reflect a pattern of adaptation (e.g., van Wingen et al., 2011). Second, although we currently have no longitudinal data on latent vulnerability in individuals who have experienced childhood maltreatment, studies of soldiers exposed to combat have reported that levels of amygdala reactivity to threat-related cues prior to combat predict subsequent levels of PTSD symptomatology (Admon et al., 2009, 2013). Third, heightened amygdala and anterior insula responsiveness to threat-related cues have been consistently associated with the clinical presentation of a number of disorders, including anxiety disorders, for which individuals who have experienced maltreatment are at increased risk.

We therefore suggest that exposure to maltreatment in childhood calibrates amygdala reactivity (including associated structures), such that it's functionality is congruent with an at-risk environment. This process of neural sensitization confers, however, a degree of latent vulnerability to future stressors or challenges, increasing the risk of anxiety and mood disorders in adolescence and into adulthood. Longitudinal studies are required to explicitly test this hypothesis.

\section{From altered threat processing to psychiatric disorder: Putative mechanisms}

The mechanism(s) by which psychiatric risk may be conferred by altered threat processing following maltreatment 
remain unclear. Here we consider several possibilities, each of which may independently contribute to risk (see Figure 1). The first is that altered vigilance to threat serves to reduce the attentional resources that would otherwise normally be allocated to other domains of functioning, including those pertinent to wider social and academic development. In other words, by privileging threat-related information, there may be a process of "attentional gating," reducing inputs from nonthreat-related domains important for optimal development in nonthreat environments.

The second is that attentional vigilance to threat leads to behaviors that shape the child's environment over time in a way that increases the likelihood of stressor experiences and decreases the likelihood of protective experiences. For example, children exposed to maltreatment may be more likely to overattribute threat in ways that increase frequency of reactive aggression; the patterns of neural response seen in children exposed to maltreatment and children with reactive (as opposed to callous-unemotional) patterns of conduct problems are remarkably similar (Viding et al., 2012). Such conflict experiences may both increase the frequency of stressor events and potentially reduce the likelihood of sustaining prosocial and supportive relationships.

The third possibility is that the latent vulnerability indexed by heightened threat reactivity results in an amplified affective response to future stressors, when those stressors are experienced and when they are subsequently processed at the cognitive level. First, exaggerated threat reactivity may lead to an amplified experience of anxiety during stressor exposure that will be more challenging for the individual to regulate (Admon, Milad, \& Hendler, 2013; McNally, 2003). Second, subsequent processing of the mental representations of traumatic stressors (both memories and cognitions), not just the actual experiences themselves, may be significantly altered by high threat reactivity. These representations, if encoded with a high threat valence, may become profoundly aversive, such that some individuals develop maladaptive strategies of repression and avoidance. However, such strategies may lead to high valence threat representations not being fully processed nor functionally integrated into a coherent self-narrative. It is thought, for example, that information-processing biases associated with threat cues lead to patterns of avoidant behavior, as well as an avoidant cognitive style, that reduces opportunities to disconfirm threatening beliefs, thus maintaining anxiety (Beck \& Clark, 1997; Heuer, Rinck, \& Becker, 2007). Heightened reactivity may therefore imbue risk not only because of an amplified response to externally perceived threat but also because it may compromise the processing of highly negative internal representations in ways that reduce the opportunities for the valence of those representations to be recalibrated.

\section{Operationalizing Latent Vulnerability: Translational Potential}

Professionals typically intervene at two stages when child maltreatment has been documented: first, to remove children from settings where they may be at risk of harm or to intervene to ensure that their home environment is made safe; and second, to provide clinical intervention if and when a child meets criteria for a psychiatric disorder. We propose that the construct of latent vulnerability has the potential to guide a third intervention stage, informing an indicated preventative approach that reduces the risk of future psychiatric disorder.

Caplan's (1964) early application of the concepts of primary, secondary, and tertiary prevention proved to be highly influential in the public health context and continue to exert influence today. These constructs, however, have in many respects been superseded by Gordon's (1983) alternative framework, which considers three categories of prevention strategies: universal, offered to the full population; selective, targeted at subpopulations identified as being at increased risk; and indicated, targeted at individuals having an increased vulnerability for a disorder but who are currently asymptomatic. These frameworks were elaborated and refined by the Institute of Medicine (Mrazek \& Haggerty, 1994), with prevention defined as those strategies designed to reduce the occurrence of new cases. The primary rationale for targeting such preventative interventions was that the potential benefit was greater than the cost or the risk of associated negative effects.

This framework provides a highly relevant context in thinking about a preventative intervention approach to children who have experienced maltreatment. A "selective prevention" approach would involve the delivery of preventative strategies to all children exposed to maltreatment with no need to screen at the individual level. This approach has the advantage of simplifying the identification and recruitment process for targeting prevention efforts with a simply defined "vulnerable population" (Springer \& Phillips, 2007). The difficulty, however, would be that such an approach would likely incur an unfeasibly high cost given the number of children affected. It may be more judicious to consider the possibility of delineating the most high-risk children in the wider group of those exposed to childhood maltreatment. In other words, it may be preferable to take an "indicated prevention" approach, which would require a process to define and identify an indicated population. In this context, we argue that successfully operationalizing the construct of latent vulnerability has the potential to identify specific individuals with known, identified risk factors that place them at elevated risk for developing a future problem or disorder. As noted earlier, such an approach would go beyond simply measuring symptoms of a disorder and would involve a focus on indices of altered neurocognitive systems thought to best index latent vulnerability.

The challenge therefore is to operationalize measures of latent vulnerability, such that a screening protocol can be developed capable of identifying an individual's level of psychiatric risk. Clearly, it is neither financially nor logistically feasible to use neuroimaging in order to conduct routine assessments of children for these purposes. Rather, we would 
suggest that the goal should be to develop behavioral and experimental tasks that provide a reliable measure of altered processing in the neurocognitive systems of interest. For example, in relation to altered threat processing, it may be possible to incorporate experimental protocols that assess attentional bias to threat as part of a clinical assessment. In our own lab, we are currently assessing a measure of attentional bias in children who have been exposed to documented maltreatment, within a longitudinal design to assess its predictive power.

\section{Challenges and Future Directions}

Here we have outlined the case for identifying latent markers of vulnerability associated with maltreatment experience that predict future psychiatric risk. At the current time, this is a theory that requires empirical evaluation. We suggest that there are five main challenges to be addressed in order to advance our understanding of latent vulnerability.

First, there is a need to investigate the potential impact of maltreatment experience on other neurocognitive systems, beyond threat processing. For example, there is evidence that maltreatment is associated with changes to memory functioning, specifically in relation to autobiographical memory. Valentino, Toth, and Cicchetti (2009) have demonstrated a pattern of overgeneral autobiographical memory in a group of children who had experienced maltreatment $(n=77)$ compared to a group of matched controls $(n=155)$. Such a pattern is of particular relevance to the question of psychiatric vulnerability given that overgeneral memory predicts later depressive symptomatology in nondepressed adults with a history of depression (e.g., Gibbs \& Rude, 2004; Mackinger, Pachinger, Leibetseder, \& Fartacek, 2000) and in adolescents at risk for depression (Rawal \& Rice, 2012). Overgeneral autobiographical memory (and potentially neural correlates of such atypical processing) may therefore represent a second candidate neurocognitive system that may index latent vulnerability. A third candidate neurocognitive system pertains to reward processing, given the emerging body of work pointing to a pattern of atypical processing of reward cues in individuals who have experienced early adversity. For example, experimental studies in humans have shown less effect of reward on task performance in children with a history of maltreatment (Guyer et al., 2006) or early caregiver deprivation (Mueller et al., 2012). Two neuroimaging studies have investigated reward anticipation in individuals who have experienced childhood adversity. Dillon et al. (2009) found reduced globus pallidus responses to reward cues in a group of young adults who had experienced maltreatment as children. Similarly, Mehta et al. (2009), using a monetary incentive delay task, found that adolescents who had experienced severe deprivation early in Romanian institutions had lower levels of ventral striatum activation during reward anticipation compared to controls. While these studies are preliminary, and are characterized by small sample sizes, they are at least suggestive that reward processing is altered following maltreatment experience. More broadly, a body of work is emerging indicating that altered reward processing may represent a core feature of several disorders associated with childhood maltreatment, including depression (Stoy et al., 2012), obsessive-compulsive disorder (Figee et al., 2011), substance misuse (Hommer, Bjork, \& Gilman, 2011), and conduct disorder (Rubia et al., 2009).

Second, although we have presented evidence for altered threat processing at the behavioral and neural levels in children and adults who have experienced maltreatment, longitudinal prospective studies are required to establish whether such indices are capable of predicting levels of future psychiatric symptomatology. To date, there remains only indirect evidence (drawn from adult studies of soldiers exposed to combat) that differences in threat processing truly index psychiatric risk, because all relevant extant studies of maltreated children have been cross-sectional in nature. While longitudinal studies are challenging, given the frequent nature of placement changes of children who are in the care system, they are essential if we are to identify accurate indices of psychiatric vulnerability. Although we postulate that latent vulnerability may best be captured by parameters that index alterations in higher order neurocognitive systems following exposure to maltreatment, it is perfectly possible that experiential (severity, duration, and timing of abuse), contextual (e.g., number of placement changes and level of social support), family (history of parental mental illness), and genetic (risk allele composites) parameters may also act as effective actuarial predictors of future risk. Longitudinal studies should assess the value of these parameters alongside indices of neurocognitive functioning, and conversely, consider those factors associated with what might be thought of as latent resilience, factors predictive of better than expected functioning. In this article, we have focused on psychiatric functioning, but as highlighted in the Introduction, maltreatment is also significantly associated with poorer educational, economic, and physical health outcomes. A further challenge of longitudinal research is to disentangle how latent vulnerability relates to these outcomes over time, either directly or via poorer psychiatric functioning.

Third, even once it is clear how best to index latent vulnerability in the laboratory, it will be necessary to determine which constructs are most sensitive and practicable in indexing such vulnerability in clinical and community settings. As discussed previously, it is unlikely that application of neuroimaging assessments will be feasible or appropriate for widescale screening. Psychological or behavioral assessments will be needed that can be administered reliably and by a range of professionals for children across different age groups. It may be possible, for example, to develop experimentally based tasks such as the well-established dot-probe task. In this paradigm, participants are first given a warning cue, followed by two words or pictures (often faces), followed by a dotprobe cue on the left or the right, in the position that was previously occupied by one of the two stimuli. The longer an individual takes to shift attention from a threat-related to 
a nonthreat-related stimulus, the greater the degree of attentional bias to threat. Typically, anxious individuals are faster to detect the presence of the dot when it is presented in the position previously occupied by the threat-related stimulus, and slower if the dot appears on the other side (Macleod \& Matthews, 1998). In addition, the degree of attention bias for masked angry faces, observed behaviorally in adolescents with clinical anxiety, has been found to be positively associated with the strength of activation in the right amygdala (Monk et al., 2008). Prospective studies of adult soldiers have also shown a relationship between atypical threat bias and future symptomatology (Wald et al., 2011). It is unlikely, however, that a single measure such as a dot-probe task will provide sufficient predictive power. We expect that a series of composite indices would be required in order to properly capture functionality of each candidate system.

Fourth, it will be important in the longer term to determine how alterations in different neurocognitive systems may additively or interactively increase risk for psychiatric disorder across the course of development. It is possible, for example, that the severity and persistence of mental health problems associated with maltreatment may be because multiple systems have adapted to, or have become calibrated in line with, specific early neglectful or maltreatment experiences. In addition, it will be important to understand how the impact of these adverse events may be moderated by either intrinsic or extrinsic protective factors, for example, individual differences in regulatory capacities or levels of social support (as illustrated in Figure 1).

Fifth, a major challenge will be to consider the form that any indicated preventative intervention would take. Such interventions would differ from existing evidence-based interventions that are aimed at targeting presenting psychiatric disorders, primarily focused on reducing the levels of current symptomatology and preventing recurrence. Indicated preventative interventions may be organized around the specific neurocognitive systems altered in maltreated individuals, for example, attention bias modification therapy in relation to threat processing (Britton et al., 2013). Such an approach would be based on the assumption of malleability and the potential of targeted individual neurocognitive systems to recalibrate. Alternatively, indicated preventative interventions could focus on enhancing more general patterns of relational functioning that may succeed in indirectly recalibrating the same systems. A series of studies drawing on attachment principles have now shown that such interventions can be very effective in improving functioning in different populations of maltreated children (e.g., Bernard et al., 2012; Cicchetti, Rogosch, \& Toth, 2006; Dozier, Peloso, Lewis, Laurenceau, \& Levine, 2008; Fisher \& Kim, 2007; Lind, Bernard, Ross, \& Dozier, 2014; Stronach, Toth, Rogosch, \& Cicchetti, 2013), and there is evidence that such approaches may even serve to normalize cortisol regulation (Cicchetti, Rogosch, Toth, \& Sturge-Apple, 2011). The attachment principles that inform these interventions represent a strong starting point in informing nondisorder-specific approaches to improve the functioning of children who are likely to show a pattern of latent vulnerability across multiple domains, and not meet criteria for any given psychiatric diagnosis. Finally, it would seem sensible that once any indicated preventative intervention was developed, it would be presented in the context of "enhancing resilience" to reduce the risk of stigmatization and to engender a genuine focus on helping the most at-risk children achieve a positive future outcome

\section{Conclusions}

In this article, we have argued for a theory of latent vulnerability. We have suggested that the experience of maltreatment and neglect in childhood can embed enduring vulnerability to psychiatric disorder by impacting on multiple neurocognitive systems during development. Changes in these systems may reflect adaptations or patterns of atypical calibration congruent with an early at-risk environment that are, however, poorly suited to more normative environmental contexts. The concept of latent vulnerability sits firmly within the framework of developmental psychopathology research, which seeks to investigate normal and abnormal, and adaptive and maladaptive, developmental processes. It is necessary to characterize what is normative if we are to understand what might be "maladaptive," from biological, psychological, and social perspectives (Cicchetti \& Toth, 2009).

Atypical development of neurocognitive systems that contribute to latent vulnerability can be thought of as hidden "stress weaknesses" in a building where the foundations have been shaped to accommodate one set of needs early in construction. However, as upper floors are added, these internal configurations may confer a weakness to the building overall, when it is exposed to future environmental stressors such as high winds or floods. It has been argued that such vulnerability is likely to emerge from the interplay of factors over time; in other words, the way in which neurocognitive systems adapt following maltreatment can set in motion interactions that increase the likelihood of disturbed behavior (Cicchetti \& Toth, 2009). For example, as outlined earlier, a child's latent vulnerability following maltreatment, as indexed by increased neural reactivity to threat, may predispose some children to greater rates of reactive aggression. Aggressive behavior may alter future social interactions, such that the child is less likely to elicit and benefit from social support, further increasing vulnerability to future psychopathology.

We have outlined how altered calibration of one candidate neurocognitive system (threat processing) may increase vulnerability to future psychopathology. The psychological and neuroimaging findings both suggest that maltreatment is associated with altered attentional allocation to threat and heightened neural responsiveness to threat cues. Such changes appear consistent with an early environment characterized by unpredictability and harm, but may become maladaptive in future more normative settings. Specifically, we speculate that altered threat processing may gate attentional 
processes constraining other developmental inputs, and augment reactivity to internal and external threat cues in ways that may amplify stress and promote patterns of avoidance increasing the likelihood of future psychiatric symptomatology. Other neurocognitive domains, however, including memory and reward processing, represent equally promising candidates for indexing latent vulnerability and warrant future enquiry. It may be the case that psychiatric disorders when they present in individuals with childhood histories of maltreatment are particularly recalcitrant because more than one system has adapted or been calibrated atypically.

\section{References}

Admon, R., Lubin, G., Rosenblatt, J. D., Stern, O., Kahn, I., Assaf, M., et al. (2013). Imbalanced neural responsivity to risk and reward indicates stress vulnerability in humans. Cerebral Cortex, 23, 28-35.

Admon, R., Lubin, G., Stern, O., Rosenberg, K., Sela, L., Ben-Ami, H., et al. (2009). Human vulnerability to stress depends on amygdala's predisposition and hippocampal plasticity. Proceedings of the National Academy of Sciences, 106, 14120-14125.

Admon, R., Milad, M. R., \& Hendler, T. (2013). A causal model of post-traumatic stress disorder: Disentangling predisposed from acquired neural abnormalities. Trends in Cognitive Sciences, 17, 337-347.

Anda, R. F., Whitfield, C. L., Felitti, V. J., Chapman, D., Edwards, V. J., Dube, S. R, et al. (2002). Adverse childhood experiences, alcoholic parents, and later risk of alcoholism and depression. Psychiatric Services, 53, 1001-1009.

Beck, A. T., \& Clark, D. A. (1997). An information processing model of anxiety: Automatic and strategic processes. Behaviour Research and Therapy, 35, 49-58.

Bernard, K., Dozier, M., Bick, J., Lewis-Morrarty, E., Lindhiem, O., \& Carlson, E. (2012). Enhancing attachment organization among maltreated children: Results of a randomized clinical trial. Child Development, 83, 623-636.

Britton, J. C., Bar-Haim, Y., Clementi, M. A., Sankin, L. S., Chen, G., Shechner, T., et al. (2013). Training-associated changes and stability of attention bias in youth: Implications for attention bias modification treatment for pediatric anxiety. Developmental Cognitive Neuroscience, 4, 52-64.

Caplan, G. (1964). Principles of preventive psychiatry. Oxford: Basic Books.

Cicchetti, D. (2013). Annual Research Review: Resilient functioning in maltreated children-Past, present, and future perspectives. Journal of Child Psychology and Psychiatry, 54, 402-422.

Cicchetti, D., \& Blender, J. A. (2006). A multiple-levels-of-analysis perspective on resilience: Implications for the developing brain, neural plasticity, and preventive interventions. Annals of the New York Academy of Sciences, 1094, 248-258.

Cicchetti, D., \& Curtis, W. (2005). An event-related potential study of the processing of affective facial expressions in young children who experienced maltreatment during the first year of life. Development and Psychopathology, 17, 641-677.

Cicchetti, D., Rogosch, F. A., \& Toth, S. L. (2006). Fostering secure attachment in infants in maltreating families through preventive interventions. Development and Psychopathology, 18, 623-649.

Cicchetti, D., Rogosch, F. A., Toth, S. L., \& Sturge-Apple, M. L. (2011). Normalizing the development of cortisol regulation in maltreated infants through preventive interventions. Development and Psychopathology, 23, 789-800.

Cicchetti, D., \& Toth, S. L. (2009). The past achievements and future promises of developmental psychopathology: The coming of age of a discipline. Journal of Child Psychology and Psychiatry, 50, 16-25.

Correll, C. M., Rosenkranz, J. A., \& Grace, A. A. (2005). Chronic cold stress alters prefrontal cortical modulation of amygdala neuronal activity in rats. Biological Psychiatry, 58, 382-391.

Creamer, M., McFarlane, A. C., \& Burgess, P. (2005). Psychopathology following trauma: The role of subjective experience. Journal of Affective Disorders, 86, 175-182.

Currie, J., \& Widom, C. S. (2010). Long-term consequences of child abuse and neglect on adult economic well-being. Child Maltreatment, 15, 111-120.
From a translational perspective, we suggest the operationalization of latent vulnerability has the potential to fundamentally reconfigure our clinical approach to childhood maltreatment.Specifically, we make the case for an indicated prevention approach, such that an emerging understanding of latent vulnerability could be operationalized in the community to identify a subgroup of children exposed to maltreatment who are at most risk of future poor outcomes. Such an approach raises the prospect of novel indicated preventative interventions that offset trajectories of psychiatric risk, reducing the likelihood that these children develop clinical disorders in the future.

Curtis, W. J., \& Cicchetti, D. (2011). Affective facial expression processing in young children who have experienced maltreatment during the first year of life: An event-related potential study. Development and Psychopathology, 23, 373.

Curtis, W. J., \& Cicchetti, D. (2013). Affective facial expression processing in 15-month-old infants who have experienced maltreatment an event-related potential study. Child Maltreatment, 18, 140-154.

Dannlowski, U., Kugel, H., Huber, F., Stuhrmann, A., Redlich, R., Grotegerd, D., et al. (2013). Childhood maltreatment is associated with an automatic negative emotion processing bias in the amygdala. Human Brain Mapping, 34, 2899-2909.

Dannlowski, U., Stuhrmann, A., Beutelmann, V., Zwanzger, P., Lenzen, T., Grotegerd, D., et al. (2012). Limbic scars: Long-term consequences of childhood maltreatment revealed by functional and structural magnetic resonance imaging. Biological Psychiatry, 71, 286-293.

Dillon, D. G., Holmes, A. J., Birk, J. L., Brooks, N., Lyons-Ruth, K., \& Piz zagalli, D. A. (2009). Childhood adversity is associated with left basal ganglia dysfunction during reward anticipation in adulthood. Biological Psychiatry, 66, 206-213.

Dozier, M., Peloso, E., Lewis, E., Laurenceau, J. P., \& Levine, S. (2008). Effects of an attachment-based intervention on the cortisol production of infants and toddlers in foster care. Development and Psychopathology, 20, $845-859$

Etkin, A., \& Wager, T. (2007). Functional neuroimaging of anxiety: A metaanalysis of emotional processing in PTSD, social anxiety disorder, and specific phobia. American Journal of Psychiatry, 164, 1476-1488.

Fales, C. L., Barch, D. M., Rundle, M. M., Mintun, M. A., Snyder, A. Z., Cohen, J. D., et al. (2008). Altered emotional interference processing in affective and cognitive-control brain circuitry in major depression. Biological Psychiatry, 63, 377-384.

Figee, M., Vink, M., de Geus, F., Vulink, N., Veltman, D. J., Westenberg, H., et al. (2011). Dysfunctional reward circuitry in obsessive-compulsive disorder. Biological Psychiatry, 69, 867-874.

Fisher, P. A., \& Kim, H. K. (2007). Intervention effects on foster preschoolers' attachment-related behaviors from a randomized trial. Prevention Science, 8, 161-170.

Gibbs, B. R., \& Rude, S. S. (2004). Overgeneral autobiographical memory as depression vulnerability. Cognitive Therapy and Research, 28, 511-526.

Gilbert, R., Widom, C. S., Browne, K., Fergusson, D., Webb, E., \& Janson, S. (2009). Burden and consequences of child maltreatment in high-income countries. Lancet, 373, 68-81.

Gordon Jr., R. S. (1983). An operational classification of disease prevention. Public Health Reports, 98, 107.

Grant, M. M., Cannistraci, C., Hollon, S. D., Gore, J., \& Shelton, R. (2011). Childhood trauma history differentiates amygdala response to sad faces within MDD. Journal of Psychiatric Research, 45, 886-895.

Guyer, A. E., Kaufman, J., Hodgdon, H. B., Masten, C. L., Jazbec, S., Pine, D. S., et al. (2006). Behavioral alterations in reward system function: The role of childhood maltreatment and psychopathology. Journal of the American Academy of Child \& Adolescent Psychiatry, 45, 1059-1067.

Harkness, K. L., Bagby, R. M., \& Kennedy, S. H. (2012). Childhood maltreatment and differential treatment response and recurrence in adult major depressive disorder. Journal of Consulting and Clinical Psychology, $80,342$. 
Harkness, K. L., \& Wildes, J. E. (2002). Childhood adversity and anxiety versus dysthymia co-morbidity in major depression. Psychological Medicine, 32, 1239-1249.

Heuer, K., Rinck, M., \& Becker, E. S. (2007). Avoidance of emotional facial expressions in social anxiety: The Approach-Avoidance Task. Behaviour Research and Therapy, 45, 2990-3001.

Hommer, D. W., Bjork, J. M., \& Gilman, J. M. (2011). Imaging brain response to reward in addictive disorders. Annals of the New York Academy of Sciences, 1216, 50-61.

Hovens, J. G. F. M., Giltay, E. J., Wiersma, J. E., Spinhoven, P., Penninx, B. W. J. H., \& Zitman, F. G. (2012). Impact of childhood life events and trauma on the course of depressive and anxiety disorders. Acta Psychiatrica Scandinavica, 126, 198-207.

Hovens, J. G. F. M., Wiersma, J. E., Giltay, E. J., Van Oppen, P., Spinhoven, P., Penninx, B. W. J. H., et al. (2010). Childhood life events and childhood trauma in adult patients with depressive, anxiety and comorbid disorders vs. controls. Acta Psychiatrica Scandinavica, 122, 66-74.

Insel, T. R. (2009). Translating scientific opportunity into public health impact: A strategic plan for research on mental illness. Archives of General Psychiatry, 66, 128-133.

Insel, T. R. (2014). The NIMH research domain criteria (RDoC) project: Precision medicine for psychiatry. American Journal of Psychiatry, 171, 395-397.

Insel, T., Cuthbert, B., Garvey, M., Heinssen, R., Pine, D. S., Quinn, K., et al. (2010). Research domain criteria (RDoC): Toward a new classification framework for research on mental disorders. American Journal of Psychiatry, 167, 748-751.

Kerestes, R., Davey, C. G., Stephanou, K., Whittle, S., \& Harrison, B. J. (2013). Functional brain imaging studies of youth depression: A systematic review. NeuroImage: Clinical, 4, 209-231.

Koenen, K. C., \& Widom, C. S. (2009). A prospective study of sex differences in the lifetime risk of posttraumatic stress disorder among abused and neglected children grown up. Journal of Traumatic Stress, 22, 566-574.

LaBar, K. S., LeDoux, J. E., Spencer, D. D., \& Phelps, E. A. (1995). Impaired fear conditioning following unilateral temporal lobectomy in humans. Journal of Neuroscience, 15, 6846-6855.

LeDoux, J. E. (2000). Emotion circuits in the brain. Annual Review of Neuroscience, 23, 155-184

Liberzon, I., \& Sripada, C. S. (2007). The functional neuroanatomy of PTSD: A critical review. Progress in Brain Research, 167, 151-169.

Liberzon, I., Taylor, S. F., Amdur, R., Jung, T. D., Chamberlain, K. R., Minoshima, S., et al. (1999). Brain activation in PTSD in response to traumarelated stimuli. Biological Psychiatry, 45, 817-826.

Lind, T., Bernard, K., Ross, E., \& Dozier, M. (2014). Intervention effects on negative affect of CPS-referred children: Results of a randomized clinical trial. Child Abuse and Neglect. Advance online publication. doi:10.1016/ j.chiabu.2014.04.004

Mackinger, H. F., Pachinger, M. M., Leibetseder, M. M., \& Fartacek, R. R. (2000). Autobiographical memories in women remitted from major depression. Journal of Abnormal Psychology, 109, 331.

MacLeod, C., \& Mathews, A. (1988). Anxiety and the allocation of attention to threat. Quarterly Journal of Experimental Psychology, 40, 653-670.

Maheu, F. S., Dozier, M., Guyer, A. E., Mandell, D., Peloso, E., Poeth, K., et al. (2010). A preliminary study of medial temporal lobe function in youths with a history of caregiver deprivation and emotional neglect. Cognitive, Affective, \& Behavioral Neuroscience, 10, 34-49.

McCrory, E. J., De Brito, S. A., Kelly, P. A., Bird, G., Sebastian, C. L., Mechelli, A., et al. (2013). Amygdala activation in maltreated children during pre-attentive emotional processing. British Journal of Psychiatry, 202, 269-276

McCrory, E. J., De Brito, S. A., Sebastian, C. L., Mechelli, A., Bird, G., Kelly, P. A., et al. (2011). Heightened neural reactivity to threat in child victims of family violence. Current Biology, 21, R947-R948.

McGlashan, T. H., \& Johannessen, J. O. (1996). Early detection and intervention with schizophrenia. Schizophrenia Bulletin, 22, 201-222.

McGorry, P. (2013). Prevention, innovation and implementation science in mental health: The next wave of reform. British Journal of Psychiatry, 202, s3-s4.

McNally, R. J. (2003). Progress and controversy in the study of posttraumatic stress disorder. Annual Review of Psychology, 54, 229-252.

Mehta, M. A., Golembo, N. I., Nosarti, C., Colvert, E., Mota, A., Williams, S. C., et al. (2009). Amygdala, hippocampal and corpus callosum size following severe early institutional deprivation: The English and Romanian
Adoptees study pilot. Journal of Child Psychology and Psychiatry, 50, 943-951.

Milad, M. R., \& Quirk, G. J. (2002). Neurons in medial prefrontal cortex signal memory for fear extinction. Nature, 420, 70-74.

Mogg, K., \& Bradley, B. P. (1998). A cognitive-motivational analysis of anxiety. Behaviour Research and Therapy, 36, 809-848.

Monk, C. S., Telzer, E. H., Mogg, K., Bradley, B. P., Mai, X., Louro, H. M. et al. (2008). Amygdala and ventrolateral prefrontal cortex activation to masked angry faces in children and adolescents with generalized anxiety disorder. Archives of General Psychiatry, 65, 568-576.

Mrazek, P. B., \& Haggerty, R. J. (Eds.). (1994). Reducing risks for mental disorders: Frontiers for preventive intervention research. Washington, DC: National Academies Press.

Mueller, S. C., Hardin, M. G., Korelitz, K., Daniele, T., Bemis, J., Dozier, M., et al. (2012). Incentive effect on inhibitory control in adolescents with early-life stress: An antisaccade study. Child Abuse and Neglect, $36,217-225$

Nanni, V., Uher, R., \& Danese, A. (2012). Childhood maltreatment predicts unfavorable course of illness and treatment outcome in depression: A meta-analysis. American Journal of Psychiatry, 169, 141-151.

Nikulina, V., Widom, C. S., \& Czaja, S. (2011). The role of childhood neglect and childhood poverty in predicting mental health, academic achievement and crime in adulthood. American Journal of Community Psychology, 48, 309-321.

Öhman, A. (2009). Of snakes and faces: An evolutionary perspective on the psychology of fear. Scandinavian Journal of Psychology, 50, 543-552.

Olds, D. L., Eckenrode, J., Henderson, C. R., Kitzman, H., Powers, J., Cole, R., et al. (1997). Long-term effects of home visitation on maternal life course and child abuse and neglect: Fifteen-year follow-up of a randomized trial. Journal of the American Medical Association, 278, 637-643.

Ozer, E. J., Best, S. R., Lipsey, T. L., \& Weiss, D. S. (2008, August). Predic tors of posttraumatic stress disorder and symptoms in adults: A metaanalysis. Psychological Bulletin, 129, 52-73.

Pape, H. C., \& Pare, D. (2010). Plastic synaptic networks of the amygdala for the acquisition, expression, and extinction of conditioned fear. Physiological Reviews, 90, 419

Pine, D. S., Mogg, K., Bradley, B. P., Montgomery, L., Monk, C. S., McClure, E., et al. (2005). Attention bias to threat in maltreated children: Implications for vulnerability to stress-related psychopathology. American Journal of Psychiatry, 162, 291-296.

Pollak, S. D., Cicchetti, D., Klorman, R., \& Brumaghim, J. T. (1997). Cognitive brain event-related potentials and emotion processing in maltreated children. Child Development, 68, 773-787.

Pollak, S. D., \& Kistler, D. J. (2002). Early experience is associated with the development of categorical representations for facial expressions of emotion. Proceedings of the National Academy of Sciences, 99, 9072-9076.

Pollak, S. D., Klorman, R., Thatcher, J. E., \& Cicchetti, D. (2001). P3b reflects maltreated children's reactions to facial displays of emotion. Psychophysiology, 38, 267-274.

Pollak, S. D., Messner, M., Kistler, D. J., \& Cohn, J. F. (2009). Development of perceptual expertise in emotion recognition. Cognition, 110, 242-247.

Pollak, S. D., \& Sinha, P. (2002). Effects of early experience on children's recognition of facial displays of emotion. Developmental Psychology, 38, 784-791.

Rawal, A., \& Rice, F. (2012). Examining overgeneral autobiographical memory as a risk factor for adolescent depression. Journal of the American Academy of Child \& Adolescent Psychiatry, 51, 518-527.

Read, J., Os, J. V., Morrison, A. P., \& Ross, C. A. (2005). Childhood trauma, psychosis and schizophrenia: A literature review with theoretical and clinical implications. Acta Psychiatrica Scandinavica, 112, 330-350.

Rieder, C., \& Cicchetti, D. (1989). An organizational perspective on cognitive control functioning and cognitive-affective balance in maltreated children. Developmental Psychology, 25, 382-393.

Rosenkranz, J. A., Venheim, E. R., \& Padival, M. (2010). Chronic stress causes amygdala hyperexcitability in rodents. Biological Psychiatry, 67, 1128-1136.

Roy, A. K., Vasa, R. A., Bruck, M., Mogg, K., Bradley, B. P., Sweeney, M., et al. (2008). Attention bias toward threat in pediatric anxiety disorders. Journal of the American Academy of Child \& Adolescent Psychiatry, 47, 1189-1196.

Rubia, K., Smith, A., Halari, R., Matsukura, F., Mohammad, M., Taylor, E., et al. (2009). Disorder-specific dissociation of orbitofrontal dysfunction in boys with pure conduct disorder during reward and ventrolateral pre- 
frontal dysfunction in boys with pure ADHD during sustained attention. American Journal of Psychiatry, 166, 83-94.

Scott, K. M., Smith, D. R., \& Ellis, P. M. (2010). Prospectively ascertained child maltreatment and its association with DSM-IV mental disorders in young adults. Archives of General Psychiatry, 67, 712-719.

Shackman, J. E., Shackman, A. J., \& Pollak, S. D. (2007). Physical abuse amplifies attention to threat and increases anxiety in children. Emotion, 7 , 838-852.

Shin, L. M., \& Liberzon, I. (2010). The neurocircuitry of fear, stress, and anxiety disorders. Neuropsychopharmacology, 35, 169-191.

Springer, J. F., \& Phillips, J. L. (2007). The Institute of Medicine framework and its implication for the advancement of prevention policy, programs and practice (Prevention Policy Paper Series). Folsom, CA: EMT Associates.

Stein, M., Simmons, A., Feinstein, J., \& Paulus, M. (2007). Increased amygdala and insula activation during emotion processing in anxiety-prone subjects. American Journal of Psychiatry, 164, 318-327.

Stoy, M., Schlagenhauf, F., Sterzer, P., Bermpohl, F., Hägele, C., Suchotzki, K., et al. (2012). Hyporeactivity of ventral striatum towards incentive stimuli in unmedicated depressed patients normalizes after treatment with escitalopram. Journal of Psychopharmacology, 26, 677-688.

Stronach, E. P., Toth, S. L., Rogosch, F., \& Cicchetti, D. (2013). Preventive interventions and sustained attachment security in maltreated children. Development and Psychopathology, 25, 919-930.

Thomas, J. L., Wilk, J. E., Riviere, L. A., McGurk, D., Castro, C. A., \& Hoge, C. W. (2010). Prevalence of mental health problems and functional impairment among active component and National Guard soldiers 3 and 12 months following combat in Iraq. Archives of General Psychiatry, 67, 614-623.

Tottenham, N., Hare, T. A., Millner, A., Gilhooly, T., Zevin, J. D., \& Casey, B. J. (2011). Elevated amygdala response to faces following early deprivation. Developmental Science, 14, 190-204.

US Department of Health and Human Services, Administration for Children and Families, Administration on Children, Youth and Families, Children's Bureau. (2013). Child maltreatment, 2012. Washington, DC: Author.

Valentino, K., Toth, S. L., \& Cicchetti, D. (2009). Autobiographical memory functioning among abused, neglected, and nonmaltreated children: The overgeneral memory effect. Journal of Child Psychology and Psychiatry, 50, 1029-1038.

van Wingen, G. A., Geuze, E., Vermetten, E., \& Fernández, G. (2011). Perceived threat predicts the neural sequelae of combat stress. Molecular Psychiatry, 16, 664-671.

Viding, E., Sebastian, C. L., Dadds, M. R., Lockwood, P. L., Cecil, C. A., De Brito, S. A., et al. (2012). Amygdala response to preattentive masked fear in children with conduct problems: The role of callous-unemotional traits. American Journal of Psychiatry, 169, 1109-1116.

Wald, I., Lubin, G., Holoshitz, Y., Muller, D., Fruchter, E., Pine, D. S., et al (2011). Battlefield-like stress following simulated combat and suppression of attention bias to threat. Psychological Medicine, 41, 699.

White, M. G., Bogdan, R., Fisher, P. M., Muñoz, K. E., Williamson, D. E., \& Hariri, A. R. (2012). FKBP5 and emotional neglect interact to predict individual differences in amygdala reactivity. Genes, Brain and Behavior, $11,869-878$.

Widom, C. S., Czaja, S. J., Bentley, T., \& Johnson, M. S. (2012). A prospective investigation of physical health outcomes in abused and neglected children: New findings from a 30-year follow-up. American Journal of Public Health, 102, 1135-1144.

Widom, C. S., Czaja, S. J., \& Paris, J. (2009). A prospective investigation of borderline personality disorder in abused and neglected children followed up into adulthood. Journal of Personality Disorders, 23, 433446.

Widom, C. S., DuMont, K., \& Czaja, S. J. (2007). A prospective investigation of major depressive disorder and comorbidity in abused and neglected children grown up. Archives of General Psychiatry, 64, 49-56.

Wiech, K., Lin, C. S., Brodersen, K. H., Bingel, U., Ploner, M., \& Tracey, I. (2010). Anterior insula integrates information about salience into perceptual decisions about pain. Journal of Neuroscience, 30, 16324-16331.

Wolfensberger, S., Veltman, D. J., Hoogendijk, W. J., Boomsma, D. I., \& de Geus, E. J. (2008). Amygdala responses to emotional faces in twins discordant or concordant for the risk for anxiety and depression. NeuroImage, 41, 544-552.

Yung, A. R., \& McGorry, P. D. (1996). The prodromal phase of first-episode psychosis: Past and current conceptualizations. Schizophrenia Bulletin, 22, 353-370. 